\title{
Structural Design and Construction of Complex-Shaped Tall Buildings
}

\author{
Kyoung Sun Moon
}

\begin{abstract}
While the early design of tall buildings culminated with the dominance of the International Style, today's pluralism in architectural design has produced tall buildings of many different forms, including more complex forms, such as twisted, tilted, tapered and free forms. This paper presents performance-based structural system design options for complex-shaped tall buildings. For each complex form category, tall buildings are designed with various structural systems, such as braced tube, diagrid and outrigger systems, and the structural performance of each system, in conjunction with the building forms and heights, is studied. Parametric structural models are generated to investigate the impacts of varying various important geometric configurations of complex-shaped tall buildings, such as the rate of twist, angle of tilt, angle of taper and degree of fluctuation of free form. The parametric models are exported to structural engineering software for analyses, design and comparative studies. While an emphasis is placed on the structural performance of complex-shaped tall buildings, architectural and construction issues are also discussed holistically.
\end{abstract}

Index Terms-Twisted tall buildings, tilted tall buildings, tapered tall buildings, freeform tall buildings, diagrids, braced tubes, outrigger structures.

\section{INTRODUCTION}

Tall buildings emerged in the late $19^{\text {th }}$ century in Chicago and New York. After decades of eclectic design in the early $20^{\text {th }}$ century, the International Style prevailed during the mid $-20^{\text {th }}$ century and produced numerous prismatic Miesian style towers all over the world. Today's architecture, including tall buildings, can be understood only through recognition of the dominance of pluralism. This contemporary architectural design trend has produced various complex-shaped tall buildings, such as twisted, tilted, tapered and freeform towers, as are the cases with the twisted Cayan Tower in Dubai, tilted Gate of Europe Towers in Madrid and tapered freeform Phare Tower in Paris. This paper studies performance-based structural system design options for various complex-shaped tall buildings.

Tall buildings carry very large gravity and lateral loads. Therefore, structural impacts of twisting, tilting, tapering and free-forming tall buildings are significant, and more careful studies are required for the design and construction of complex-shaped tall buildings. Though not uncommon these days, complex-shaped tall buildings are a still very recent architectural phenomenon, and only a limited amount of

Manuscript received March 15, 2014; revised May 27, 2014

Kyoung Sun Moon is with Yale University School of Architecture, New Haven, CT 06511 USA (e-mail: kyoung.moon@yale.edu). related research has been conducted. This paper systematically studies and comparatively evaluates different structural systems employed for various complex-shaped tall buildings. Construction issues are also discussed holistically.

\section{Method of Design AND ANALYsis}

Tall buildings of various complex forms and heights are designed with today's prevalent tall building structural systems, such as diagrids, braced tubes and outrigger systems, and their comparative structural performances are studied. Considering that the structural design of tall buildings is generally governed by lateral stiffness rather than strength [1], stiffness-based design methodologies are used to design the tall building structures of various complex forms.

Preliminary structural member sizes for conventional rectangular box-form towers of diagrid, braced tube and outrigger systems are generated first to satisfy the maximum lateral displacement requirement of a five hundredth of the building height. The studied conventional box-form tall buildings' plan dimensions are $36 \mathrm{~m}$ x $36 \mathrm{~m}$, with an $18 \mathrm{~m} \mathrm{x}$ $18 \mathrm{~m}$ core at the center and typical story heights of $3.9 \mathrm{~m}$. based on these dimensions, the height-to-width aspect ratios of the studied 60-, 80- and 100-story buildings are 6.5, 8.7 and 10.9 , respectively. Core structures are designed to carry only gravity loads for the tube type structures, such as diagrids and braced tubes. For outrigger structures, core structures are designed as braced frames to carry both gravity and lateral loads. The SEI/ASCE Minimum Design Loads for Buildings and Other Structures is used to establish the wind load. The structures are assumed to be in Chicago and within category III, which implies that there is a substantial hazard to human life in the event of failure.

Once the structural design and analyses of the rectangular box form tall buildings are completed, comparable complex-shaped tall buildings of each form category are designed with diagrids, braced tubes and outrigger structures. For twisted, tilted, tapered and freeform tall buildings, parametric structural models are generated using Rhino/Grasshopper to investigate each system's structural performance depending on the rate of twist, angle of tilt, angle of taper and degree of fluctuation of free form. The models are exported to structural engineering software, SAP 2000 , for design, analyses and comparative studies. In order to comparatively estimate the structural performances of various structural systems employed for twisted, tilted, tapered and freeform structures, the preliminary structural member sizes determined for the conventional box form towers are also used for the complex-shaped tall buildings with some minor adjustments when necessary. 


\section{Twisted TALl Buildings}

Employing twisted forms for tall buildings is a recent architectural phenomenon. Twisted forms employed for today's tall buildings can be understood as a reaction to rectangular box forms of modern architecture. In fact, this contemporary architectural phenomenon is not new in architecture. It is comparable to twisted forms of Mannerism architecture at the end of Renaissance architecture. For example, in Cortile della Cavallerizza at Palazzo Ducale in Mantua, Giulio Romano designed twisted columns. Twisted forms can be found again in today's tall buildings, such as the Shanghai Tower in Shanghai designed by Gensler (Fig. 1), Infinity Tower in Dubai by SOM, and Chicago Spire project in Chicago by Calatrava.

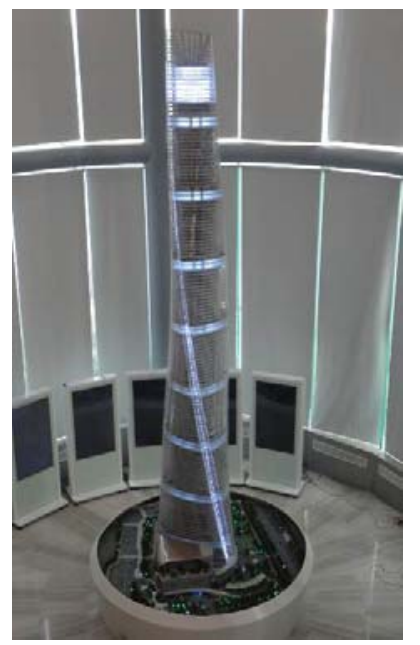

Fig. 1. Shanghai tower model (courtesy of the author).

In terms of static response, twisted forms are not structurally beneficial. Fig. 2 shows prismatic and twisted towers with a square plan. If solid towers are considered, the moment of inertia of a square plan does not change regardless of its twisted angle. However, if building type structures composed of many frame members are considered, the lateral stiffness of the twisted tower is smaller than that of the straight tower [2].

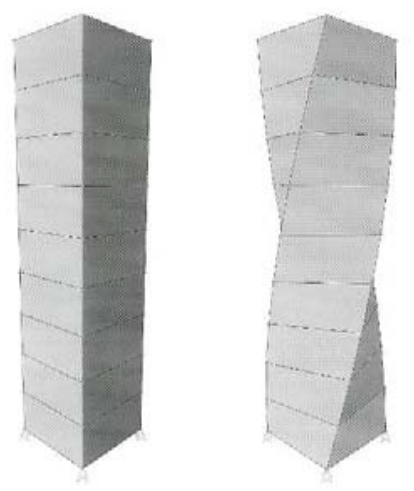

Fig. 2. Prismatic vs. twisted tower.

Both diagrids and braced tubes are very efficient structural systems for tall buildings of conventional shapes, such as rectangular box form towers. If these structural systems are employed for twisted tall buildings, the systems' lateral stiffness decreases as the rate of twist increases. The stiffness reduction of braced tubes, composed of verticals and diagonals, is much more sensitive to the rate of twist, compared to that of diagrids, composed of only diagonals.
And this sensitivity is accelerated as the building height increases. Fig. 3 clearly shows this phenomenon with the maximum lateral displacements of twisted diagrids and braced tubes of various heights and rates of twist.

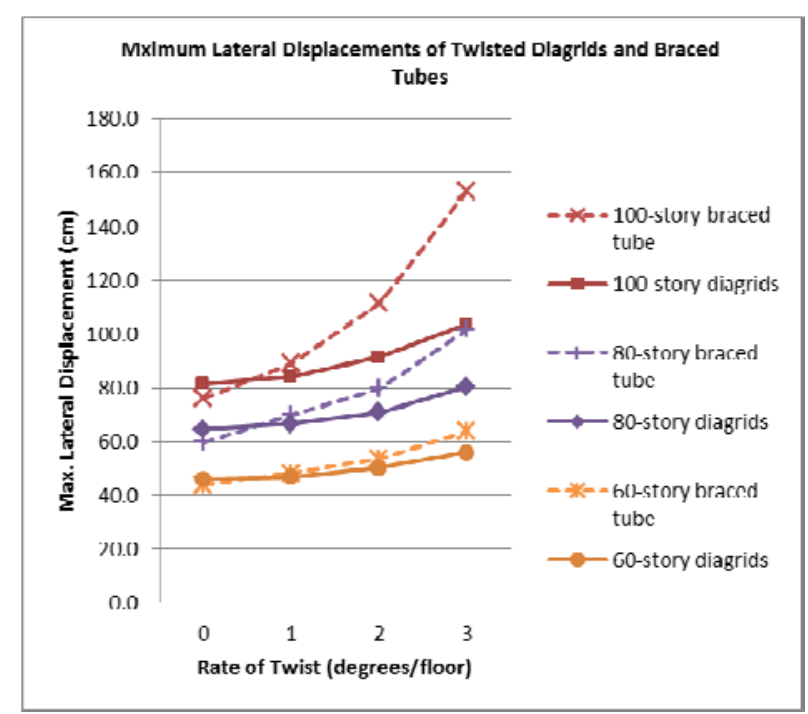

Fig. 3. Maximum lateral displacements of twisted diagrids and braced tubes.

Lateral load-carrying mechanism of outrigger structures is different from that of tube type structures, such as diagrids and braced tubes. Both lateral shear forces and overturning moments are carried by perimeter diagrids and braced tubes in the perimeter tube type structures [3], [4]. In outrigger structural systems with braced core structures, the braced cores carry lateral shear forces and a portion of overturning moments. Perimeter mega-columns connected to the stiff braced core structure through outrigger trusses also significantly contribute to the bending rigidity in the outrigger structural system [5].

As the outrigger structure is twisted, the perimeter mega-columns wrap around the building spirally. Lateral stiffness of the outrigger structures with these spirally slanted perimeter mega-columns is substantially reduced as the rate of twist increases. Fig. 4 shows an example of twisted outrigger structures, and Fig. 5 summarizes the maximum lateral displacements of twisted outrigger structures of various heights and rates of twist. It clearly shows the decreased lateral stiffness of the twisted outrigger structures with the increased rate of twist.

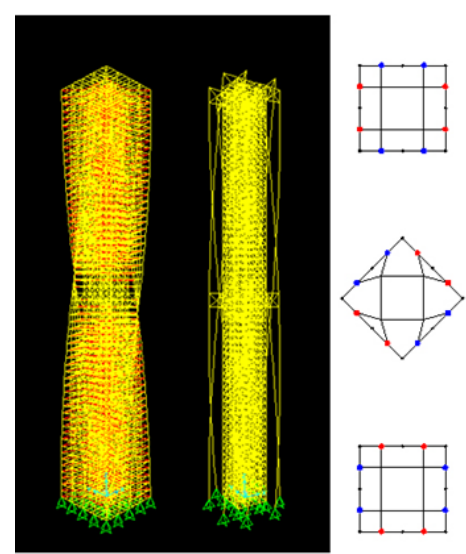

Fig. 4. 60-story twisted outrigger structures with a rate of twist of 1.5 degrees/floor.

Considering the substantial stiffness reduction caused by 
the spirally slanted mega-columns, outrigger structures with setback vertical mega-columns may be a feasible design alternative to enhance constructability. The Chicago Spire project of twisted form employs the outrigger structure with setback vertical mega-columns.

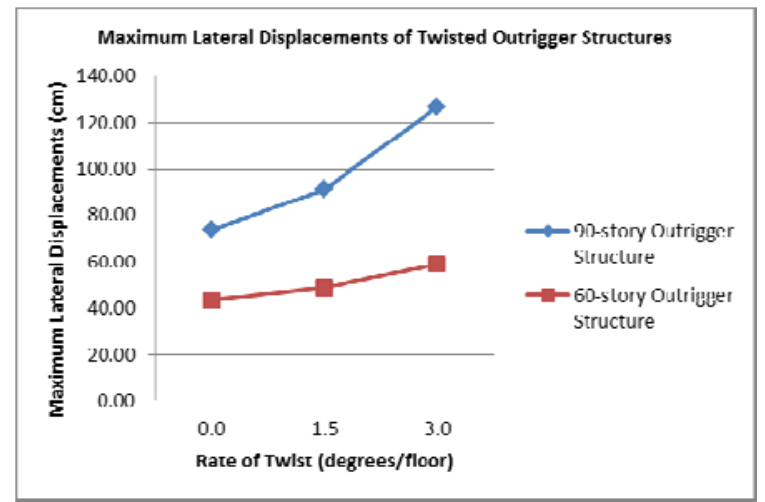

Fig. 5. Maximum lateral displacements of twisted outrigger structures.

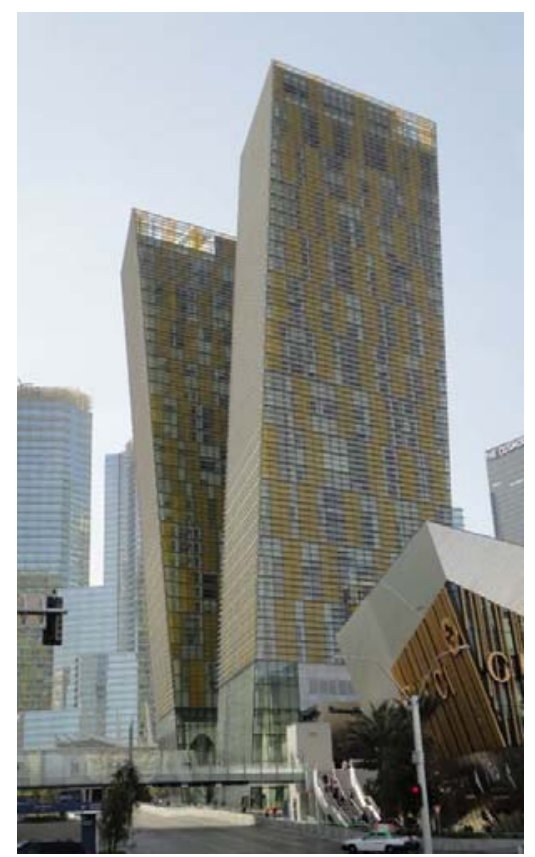

Fig. 6. Veer tower in Las Vegas (courtesy of the author).

With regard to the across-wind direction dynamic responses due to vortex shedding, it should be noted that a twisted tower generally performs better than a comparable prismatic one, as it can mitigate wind-induced vibrations by disturbing the formation of organized alternating vortexes. Considering the fact that the vortex-shedding-induced lock-in phenomenon often produces the most critical structural design condition for tall buildings [6], twisted building form's structural contribution can be significant.

\section{Tilted TAll BuILDingS}

Buildings have traditionally been constructed vertically, orthogonal to the ground. When a building is found to be tilted, it is typically an indication of some serious problems occurred to the building. The leaning Tower of Pisa is a famous example of tilted buildings due to differential settlements. Today, however, tilted buildings are intentionally designed and built to produce more dramatic architecture, as are the cases with the Gate of Europe Towers of 1996 in Madrid designed by Philip Johnson/John Burgee, Veer Towers of 2010 in Las Vegas by Helmut Jahn (Fig. 6), and the design of the Signature Towers in Dubai by Zaha Hadid.

The structural performance of a tilted tall building is dependent upon its structural system and angle of tilt. Fig. 7 shows example tilted tall buildings. Compared to the perimeter tube type structures, such as braced tubes and diagrids, the outrigger system provides greater lateral stiffness for tilted towers because of the triangulation of the major structural components - the braced core, outrigger trusses and mega-columns - caused by tilting the tower, as can be seen in Fig. 7.
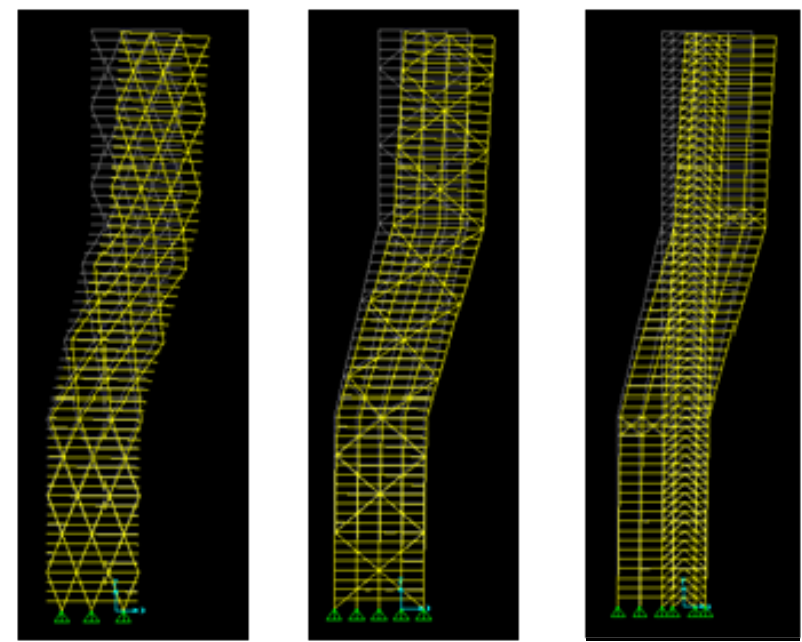

Fig. 7. Tilted diagrids (elevation view), braced tube (elevation view) and outrigger structure (section view).

Sixty story tilted tall buildings of various structural systems are studied in this section. The angles of tilt studied are 4, 7, 9 and 13 degrees, which correspond to offsets of 0 , 12,16 and 20 stories, respectively, at both top and bottom of the structure. Fig. 8 shows braced tube structures with these angles of tilt. Diagrids and outrigger structures are also tilted with these angles. Fig. 9 summarizes wind-induced maximum lateral displacements of the 60 -story tilted braced tubes, diagrids and outrigger structures. The lateral stiffness of the braced tube and diagrid systems is not substantially influenced by the angle of tilt between 0 and 13 degrees studied here. The lateral stiffness of the outrigger system is even increased by tilting the tower due to the triangulation of the major structural components - the braced core, mega-columns and outrigger trusses.

Tilted tall buildings are subjected to significant initial lateral deformations due to eccentric gravity loads. Gravity-induced lateral displacements increase as the angle of tilt increases in all the three structural systems. Among them, the outrigger structures produce relatively small gravity-induced lateral displacements again because of the triangulation of the major structural components. These gravity-induced deformations can be managed substantially through careful construction planning.

As the angle of tilt increases, very large localized stresses are developed in tilted tall buildings. Though structural design of tall buildings is generally governed by lateral 
stiffness, careful studies on satisfying strength requirements are also essential for tilted tall buildings. Large tensile forces, not very often found in conventional vertical tall buildings, can be developed in tilted tall buildings. Careful design studies on the connections of the tensile members of tilted tall buildings are required.

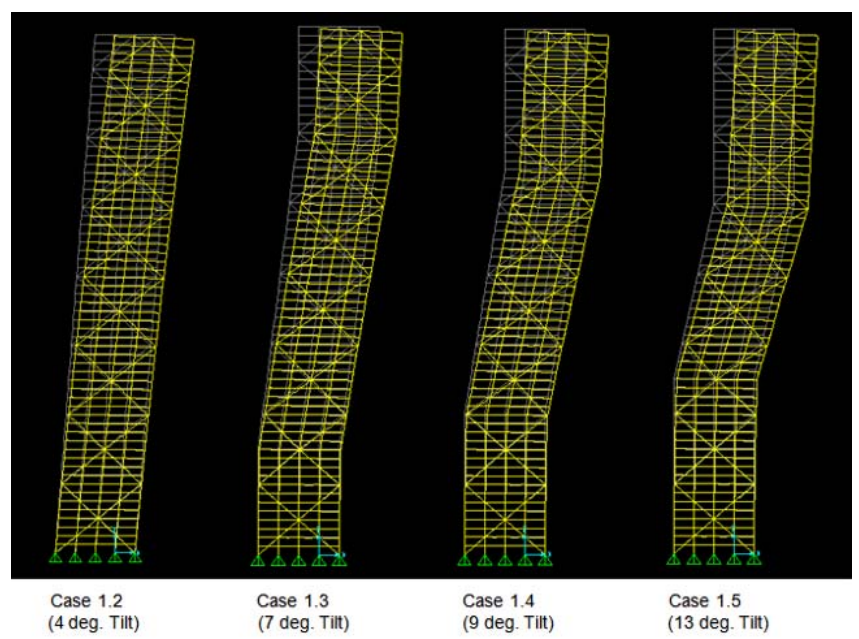

Fig. 8. Tilted braced tubes with angles of tilt of 4, 7, 9 and 13 degrees.

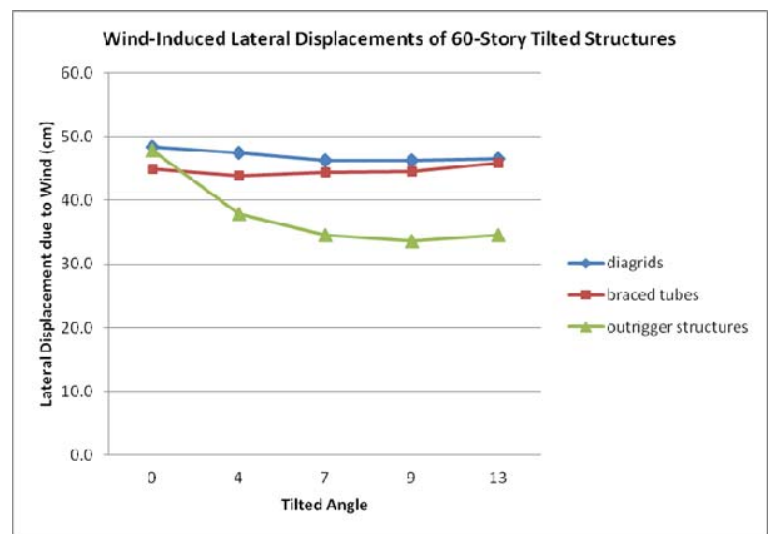

Fig. 9. Wind-induced lateral displacements of 60-story tilted tall buildings.

\section{TAPERED TALl BUILDINGS}

Compared to prismatic forms, tapered forms provide many advantageous aspects for structural systems for tall buildings. The magnitudes of shear and overturning moments due to lateral forces grow toward the base of the building, and, as was discussed earlier, for very tall buildings, it is common that lateral loads rather than gravity loads govern the structural design. In order to resist lateral loads, tapered forms with greater building width toward the base are more desirable than prismatic forms because tapered form helps increase the lateral stiffness and reduce lateral loads.

Tapered forms also help tall buildings prevent shedding organized alternating vortices, which can cause the lock-in condition, along the building height. Therefore, tapered tall buildings are less susceptible to severe across-wind direction vibrations caused by vortex-shedding than prismatic tall buildings.

Further, tapered forms are often more desirable architecturally for mixed-use tall buildings [7]. For residential functions in tall buildings, for example, it is important to make living space not too far away from natural light to maximize comforts for occupants. For commercial office functions, however, natural light is less important and deeper rentable space is often desired. Therefore, tapered tall buildings, with commercial office functions on the lower levels and residential functions on the higher levels, work very well architecturally. A famous example of this type of spatial organization in a tapered tall structure can be found in the 100-story tall John Hancock Center of 1969 in Chicago (Fig. 10), [8].

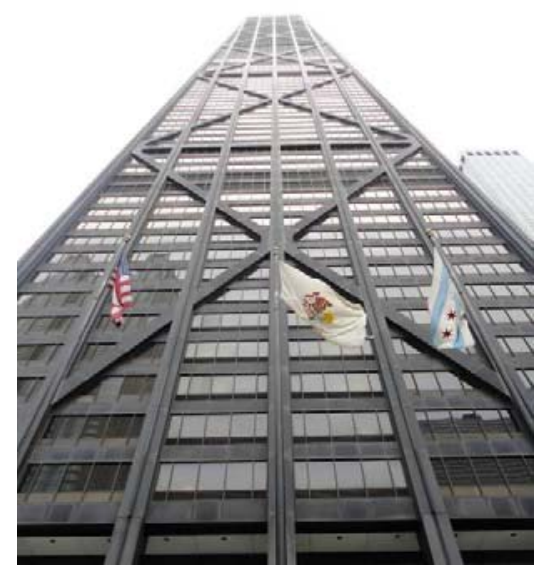

Fig. 10. John hancock center in chicago (courtesy of the author).

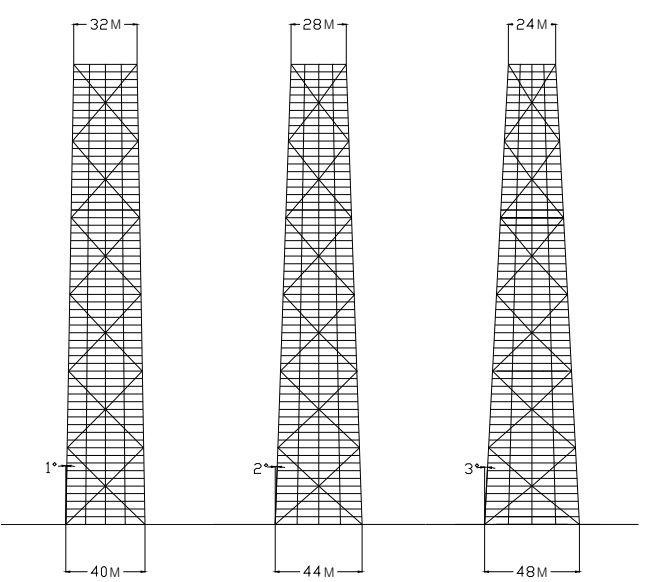

Fig. 11. Tapered braced tube structures.

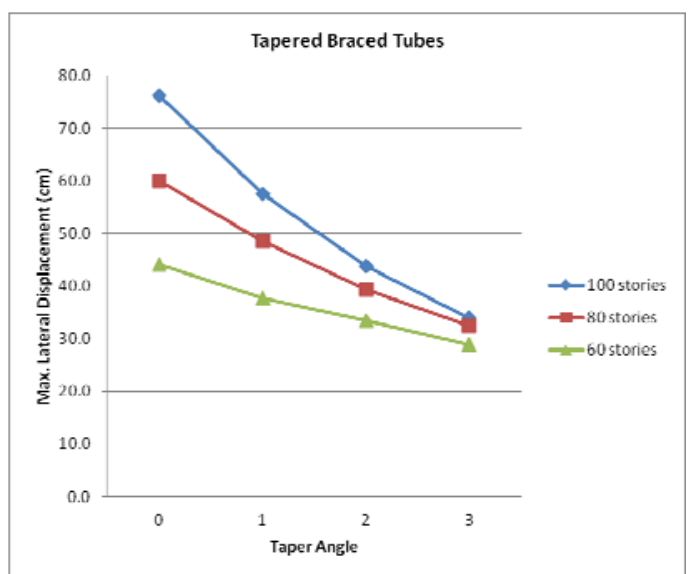

Fig. 12. Wind-induced lateral displacements of 60-story tapered tall buildings.

The lateral performance characteristics of tapered perimeter tube type structures are very similar. As the angle of taper increases, the lateral stiffness of diagrids and braced tubes is substantially increased. Fig. 11 shows 60-story tapered braced tubes with angles of taper of 1,2 and 3 degrees. Each building is designed to have the identical gross 
floor area regardless of the different angles of taper. Fig. 12 summarizes the maximum lateral displacements of the tapered braced tubes of 60,80 and 100 stories. As the building height is increased, the impact of taper in terms of reducing lateral displacement becomes greater due to the combined effect of increased stiffness and reduced lateral loads. The result of the same study with diagrid structures is very similar.

The performance characteristics of tapered outrigger structures are somewhat different from those of tapered braced tubes and diagrids. The stiffness of the lower level outrigger trusses, which connect the mega-columns and braced core, is reduced as the building is tapered because their length is increased. This makes the lateral performance of tapered outrigger structures different from that of the perimeter tube type structures. However, the lateral stiffness of outrigger structures is still increased substantially, as the angle of taper is increased.

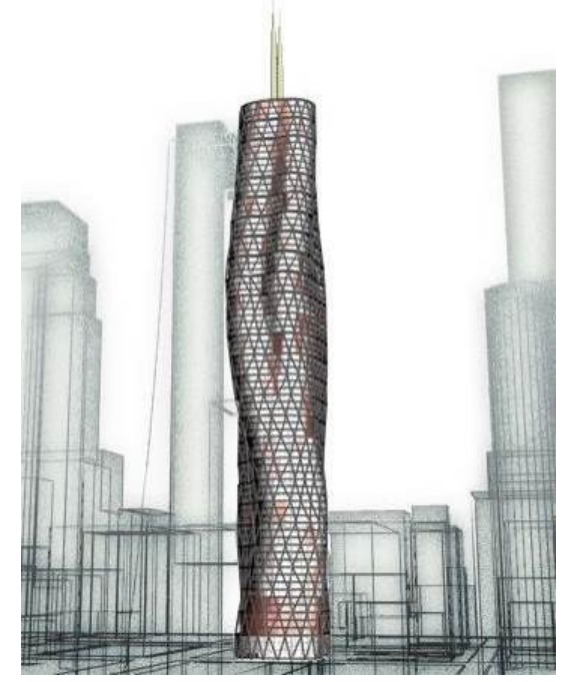

Fig. 13. Diagrid freeform tall building (courtesy of nicholas georgaklis and adam gimpert).

\section{FREEFORM TALL BUILDINGS}

The number of freeform tall building projects has been rapidly increasing these days. In the past, only a few freeform tall building projects were proposed, such as the Max Reinhardt Haus project of 1992 by Peter Eisenman and the New York Time Building proposal of 2000 by Frank Gehry. It was a very difficult task to perform the structural design and analysis of irregular freeform tall buildings in the past. Today, however, with the development of structural design/analysis computer software, many freeform tall structures are designed and actually constructed [9]. Relying on the powerful support of contemporary structural engineers, some architects find their design solutions in free forms feasible. These architects include Daniel Libeskind, Zaha Hadid and Thom Mayne to name a few.

Even though the supporting structural systems behind the free forms vary depending on the project-specific situations, diagrids are often employed as primary structural systems for freeform tall buildings, as can be observed from the Fiera Milano Tower in Milan by Daniel Libeskind and the Phare Tower in La Defense by Thom Mayne. As building form becomes more irregular, finding an appropriate structural system for better performance and constructability is essential to successfully carry out the project. The diagrid structural system has great potential to be developed as one of the most appropriate structural solutions for irregular freeform towers. Triangular structural geometric units naturally defined by diagrid structural systems can specify any irregular freeform tower more accurately without distortion. Fig. 13 shows a freeform tall building designed with digrids.

Diagrid systems are employed for 60-story freeform tall buildings to investigate their structural performance. Freeform geometries are generated using sine curves of various amplitudes and frequencies. For the purpose of comparison, preliminary member sizes for the 60-story conventional rectangular box form diagrid tall building are generated first to satisfy the maximum lateral displacement requirement of a five hundredth of the building height.

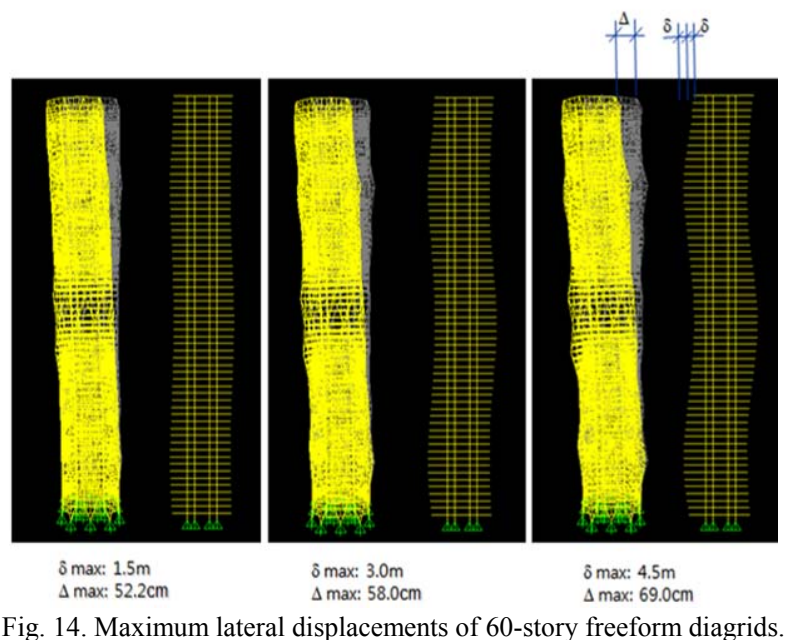

Compared to the rectangular box form diagrid structure, which has $36 \times 36$ meter square plan on each floor, the floor plans of the first freeform case shown in Fig. 14 fluctuate within the $+/-1.5$ meter boundaries of the original square. The floor plans of the second and third cases fluctuate within the $+/-3$ and 4.5 meter boundaries of the original square respectively. Each building is designed to have the identical gross floor area regardless of these geometry changes.

As can be seen in Fig. 14, which shows the deformed shape of each diagrid structure in a scale factor of 20, the lateral displacement of the structure becomes larger as the freeform shape deviates more from its original rectangular box form. This is much related to the change of the diagrid angle caused by free-forming the tower. The straight tower designed first for the comparison is configured with the optimal diagrid angle of about 70 degrees. As the degree of fluctuation of freeform increases, the diagrid angle deviates more from its original optimal condition, which results in substantially reduced lateral stiffness of the tower. Therefore, freeform shapes should be determined with careful considerations of not only architectural but also structural performance.

With regard to the across-wind direction dynamic responses due to vortex shedding, irregular free forms also help tall buildings prevent shedding organized alternating vortices, which can cause the lock-in condition, along the 
building height. Therefore, freeform tall buildings are generally less susceptible to severe across-wind direction vibrations than prismatic tall buildings.

\section{CONCLUSION}

Today's pluralism in architecture has produced many tall buildings of complex forms. This paper presented comparative lateral performance of diagrids, braced tubes and outrigger structures, employed of twisted, tilted, tapered and freeform tall buildings.

Lateral stiffness of diagrids, braced tubes and outrigger structures is reduced when they are employed for twisted tall buildings. As the rate of twist is increased, the rate of stiffness reduction is also increased.

Lateral stiffness of tilted diagrids and braced tubes is not substantially influenced by the angle of tilt ranging from 0 to 13 degrees studied in this paper. Lateral stiffness of outrigger structures is somewhat increased as they are tilted because of the triangulation of the major components of the lateral load resisting system - the braced core, mega-columns and outrigger trusses. Tilted tall buildings are laterally deformed by not only wind loads but also eccentric distribution of gravity loads. The gravity-induced lateral displacements can be substantially managed through careful construction planning.

Tapered form is typically advantageous for tall building structures. As the angle of taper is increased, the lateral stiffness of the structural system is increased and the wind loads applied to the structure is decreased. Consequently, the lateral displacements of tapered tall buildings are substantially decreased compared to comparable prismatic tall buildings. Tapered form also works well architecturally for tall buildings with commercial office functions on the lower levels and residential functions on the higher levels.

For freeform tall buildings, the diagrid structural system has great potential to be developed as one of the most appropriate structural solutions because triangular structural geometric units naturally defined by diagrids can specify any irregular freeform tower more accurately without distortion. Lateral stiffness of diagrid structural systems employed for freeform tall buildings is decreased. As the degree of fluctuation of free form is increased, the rate of lateral stiffness reduction is also increased.

Vortex-shedding-induced lock-in phenomenon often produces the most critical structural design condition for tall buildings. Regarding the across-wind direction dynamic responses, complex-shaped tall buildings generally perform better than comparable prismatic tall buildings, as they can mitigate wind-induced vibrations by disturbing the formation of organized alternating vortexes.

Today's complex-shaped tall buildings require more complicated system design, analysis and construction. Not only architectural but also structural and other related performance issues should be considered holistically to produce higher quality built environments. Well-organized coordination between architects and engineers is essential.

\section{ACKNOWLEDGMENT}

This research is part of the Korea Super-Tall Building R\&D Project (VC-10) supported by High-Tech Urban Development Program of the Ministry of Land, Transport and Maritime Affairs of Korea.

\section{REFERENCES}

[1] J. J. Connor, Introduction to Structural Motion Control, New York: Prentice Hall, 2003.

[2] M. M. Ali and K. Moon, "Structural developments in tall buildings: Currents trends and future prospects," Architectural Science Review, vol. 50, no. 3, pp. 205-223, 2007.

[3] K. Moon, "Stiffness-based design methodology for steel braced tube structures: A sustainable approach," Engineering Structures, vol. 32, pp. 3163-3170, 2010.

[4] K. Moon, J. J. Connor, and J. E. Fernandez, "Diagrid structural systems for tall buildings: characteristics and methodology for preliminary design," The Structural Design of Tall and Special Buildings, vol. 16, no. 2, pp. 205-230, 2007.

[5] B. Smith and A. Coull, Tall Building Structures: Analysis and Design, New York: Wiley, 1991.

[6] E. Simiu and R. H. Scanlan, Wind Effects on Structures: Fundamentals and Applications to Design, 3rd Edition, New York: Wiley, 1996.

[7] I. Abalos and J. Herreros, Tower and Office: From Modernist Theory to Contemporary Practice, Cambridge: MIT Press, 2003.

[8] R. Kowalczyk, R. Sinn, and M. B. Kilmister, Structural Systems for Tall Buildings, presented at council on Tall Buildings and Urban Habitat Monograph, New York: McGraw-Hill, 1995.

[9] L. S. Beedle, M. M. Ali, and P. J. Armstrong, The Skyscraper and the City, Ceredigion: Edwin Mellen Press, 2007.

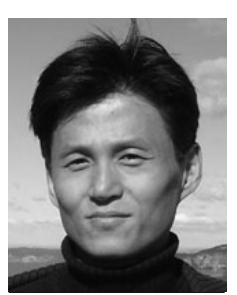

Kyoung Sun Moon is an associate professor at Yale University School of Architecture. He received his B.S. from Seoul National University and an M.S.C.E. from the University of Illinois at Urbana-Champaign, and a Ph.D. from Massachusetts Institute of Technology. His background includes work at Skidmore, Owings, and Merrill in Chicago and the Republic of Korea Navy.

Moon's primary research area is integration between the art and science/technology of architecture, with a focus on tall buildings. His research articles on tall buildings have appeared in many scholarly journals such as The Structural Design of Tall and Special Buildings, Engineering Structures, Journal of Architectural Engineering, Architectural Science Review, Journal of Urban Technology, and International Journal of Sustainable Building Technology and Urban Development. Dr. Moon has also presented research papers on tall buildings in numerous relevant international conferences including the 8 th and $9^{\text {th }}$ Council on Tall Buildings and Urban Habitat World Congresses held in Dubai and Shanghai, respectively.

Moon is a licensed architect in the U.S., and a member of the American Institute of Architects, American Society of Civil Engineers Committee on Tall Buildings, and Council on Tall Buildings and Urban Habitat Expert Review Panel. He is an editorial board member of scholarly journals such as the International Journal of High-Rise Buildings and Journal of Architectural Engineering Technology. 